\title{
An $a b$ initio study of surface electrochemical disproportionation: the case of a water monolayer adsorbed on a Pd(111) surface
}

\author{
Jean-Sébastien Filhol*, Marie-Liesse Doublet \\ Institut Charles Gerhardt Montpellier \\ UMR 5253 CNRS-UM2-ENSCM-UM1 \\ CTMM, Université Montpellier 2, \\ Place Eugène Bataillon, 34095 Montpellier Cédex 5 - France \\ Keywords: water monolayer, $\operatorname{Pd}(111)$ surface, disproportionation, electrochemistry, \\ Density Functional Theory, stability diagram, Fukui function, orbital analysis.
}

\begin{abstract}
We present a theoretical study of the structural response of model water monolayers on a Pd(111) surface upon various electrochemical conditions. Whereas the water molecules pointing toward the vacuum are mostly electrochemically inactive, those lying parallel to the surface undergo an oxidative adsorption under oxidizing conditions, and those pointing toward the surface show a reductive adsorption under reducing conditions. The oxidative adsorption is shown to result from the interaction of the $\mathrm{H}_{2} \mathrm{O} 1 b_{1}$ orbital with the metal surface $\mathrm{dz}^{2}$ band which becomes bonding only under oxidative conditions. The reductive adsorption arises from the interaction of the $\mathrm{H}_{2} \mathrm{O} 4 a_{1}-2 b_{2}$ orbitals with the $d z^{2}$ band of the Pd surface. This electronic analysis of electrochemical effects is further validated using the Fukui function associated with the water monolayers. The Fukui function is shown to be a powerful tool for studying electrochemical effects as it is directly linked with the electrochemical bond reorganization and other parameters like the surface capacitance. The electrochemical stability diagram for these water monolayers is computed using a new correction for the DFT electrochemical calculations and compared to previous results. The phase transformation from a positively charge $\mathrm{H}$-up to a negatively charged $\mathrm{H}$-down phase is here confirmed with, however, a potential shifted up to $4.5 \mathrm{~V}$ compared to our previous report (4.3 V). The neutral phase is found to consist in a positively charged $\mathrm{H}$-up phase and a negatively charged $\mathrm{H}$-down phase. This phenomenon is confirmed by large super-cell calculations with different ratio of $\mathrm{H}$-up and $\mathrm{H}$-down phases. The stability of the mixed charged phases is then rationalized in terms of an electrochemical disproportionation whose origin and consequences are further discussed in regards to the results previously obtained on the basis of the ice rules.
\end{abstract}

\footnotetext{
* Corresponding author: filhol@univ-montp2.fr
} 


\section{Introduction}

The water-solvent interface is extremely important for various fields of surface science electrochemistry ${ }^{1,2}$ and technologies such as catalysis, corrosion, electroplating, fuel-cells, Li-lon batteries or biocompatible materials. Despite decades of research ${ }^{3,4}$, the full complexity of these interfaces was not unraveled and debates still exist on the driving forces governing the water molecules organization on a metal surface ${ }^{5-10}$. One major reason for such difficulties is the very different interactions water molecules may exhibit with metal surfaces, depending on the transition metal considered. ${ }^{3,11,12}$ Water molecules have a strong dipolar moment that interacts with local electric fields, create strong network of $\mathrm{H}$-bonds with the surrounding water molecules constraining efficiently their relative geometries, have non negligible dispersive interactions, ${ }^{13}$ transform into different structures with the applied potential, ${ }^{14,15}$ can be both oxidized or reduced upon electrochemical conditions, ${ }^{2}$ can dissociate on certain metal ${ }^{5}$ (e.g. Ru(0001)), can stay intact on other metal $^{6,15}$ (e.g. $\left.\mathrm{Pd}(111)\right)$, can be involved in proton transfer and possibly have strong hydrogen nucleus quantum effect ${ }^{16}$. Experimentally, these multiple phenomena result in an extremely complex behavior which crucially depends on coverage, temperature ${ }^{17,18}$ or applied surface biased. ${ }^{14,19,20}$ Modeling such complex phenomena is a true challenge from the computational point of view, in particular to account for fine electronic, electrostatic and electrochemical effects. The simplest models to describe a metal-water interface is the ordered water monolayer that was intensively studied both from theoretical $\left.\right|^{5-8,17,21}$ and experimental ${ }^{9,22}$ sides. Despite their simplicity, these monolayers have specific and complex structures and behaviors. In a recent study, ${ }^{18}$ Tatarkhanov et al. have used scanning tunneling microscopy (STM) to show that a water monolayer adsorbed on $\mathrm{Pd}(111)$ or $\mathrm{Ru}(0001)$ surfaces exhibits a much more complex structure than the ideal "ice-like" structure. Using first-principles DFT calculations, they have proposed new extended water structures, built on the so-called ice rules that could match the experimental STM images. While this study gives a consistent picture of the water organization over the metal surface, it nevertheless requires large super-cell calculations and do not allow to fully understand the origin of such water organization. Indeed, based on a purely geometrical assumption, the ice-rule states that water should adopt geometries that maximize the number of $\mathrm{O}-\mathrm{Metal}$ and $\mathrm{H}-\mathrm{OH}_{2}$ bonds, thus neglecting the bond strength dependence to the environment. Though, in recent papers, ${ }^{2,15}$ we have used an electrochemical approach within the framework of density functional theory (DFT) to show that electrochemical effects could drastically affect the water surface organization. Applied to the water- 
$\operatorname{Pd}(111)$ interface, this study shows that a globally neutral mixture of positively and negatively charged $\mathrm{H}_{2} \mathrm{O}$ phases can be more stable than the purely neutral surfaces previously reported, ${ }^{9}$ due to the modification of the $\mathrm{H}_{2} \mathrm{O}$-metal bond strength. This suggests that water organization on $\mathrm{Pd}$ surface is not only governed by the ice-rule, but also tuned by electrochemical effects, even when neutral interface are considered. The aim of this paper is to provide a complete analysis of neutral and charged $\mathrm{H}_{2} \mathrm{O} / \mathrm{Pd}(111)$ interfaces, by combining first-principles DFT calculations with orbital analysis. In order to extract a clear and consistent picture of the electrochemical effects on water organization, two simple models (hereafter denoted $\mathrm{H}$-up and $\mathrm{H}$-down) have been chosen. They consist in monolayers made of water molecules lying parallel to the surface, and some others being tilted from the surface plane with an $\mathrm{O}-\mathrm{H}$ bond pointing toward either the surface or the vacuum.

To capture the leading electrochemical effects at the $\mathrm{H}_{2} \mathrm{O} / \mathrm{Pd}(111)$ interfaces (neutral and charged), we followed the electrochemical DFT approach initially proposed by Filhol and Neurock ${ }^{2}$ and further improved by Mamatkulov and Filhol. ${ }^{23}$ First, we focused on the structural change occurring in the model water structures as a function of the applied potential. This study shows that water molecules with different orientations toward the surface behave differently upon oxidizing/reducing conditions. Then, we focused on the electronic factors governing the structural modification using an orbital analysis as a function of the potential. This analysis is then correlated to the Fukui functions associated with the different water structures. Then the electrochemical stability diagram for the water monolayer is computed using the improved approach mentioned above and compared to previous results. While leading mostly to an equivalent electrochemical phase transformation than the one previously reported, ${ }^{15}$ the equilibrium potential is shifted to higher potential ( $4.5 \mathrm{~V}$ ) and associated with charge exchange of $0.26 \mathrm{e}$ between oxidant and reducer phases. The neutral surface is then shown to results from a nearly 50-50 mixture of positively charged $\mathrm{H}$-up and negatively charged $\mathrm{H}$-down phases. This phenomenon is validated using a 135 atoms neutral super-cell built on $\mathrm{H}$-up and $\mathrm{H}$-down subunits with various ratios. The stability of the mixed charged phases is then confirmed and associated to the synergic formation of stronger bonds in the charged sub-parts of the interface. The decomposition of a neutral water layer into charged domains of $\mathrm{H}$-up and $\mathrm{H}$-down phases is interpreted in terms of an electrochemical disproportionation. The origin and the consequences of such an effect are discussed in particular to understand the experimental structures. 


\section{Methodology}

Electronic structure calculations were performed within the density-functional theory (DFT) framework using the $\mathrm{PBE}^{24}$ generalized gradient approximation and projector augmented wave $(\text { PAW })^{25}$ pseudo-potentials, as implemented in the VASP code. ${ }^{26-28}$ Following our previous report, ${ }^{15}$ the $\operatorname{Pd}(111)$ surface was modeled using a super-cell consisting in a 5-layer slab (with the calculated in-plane lattice constant $2.80 \AA$ ) and a vacuum layer exceeding $15 \AA$. The water monolayer was symmetrically adsorbed on both sides of the slab to cancel out most of the unphysical electrostatic interactions between periodic images in the z-direction. Two-dimensional $(\sqrt{3} \times \sqrt{3})$ and $\left(\begin{array}{cc}8 & -1 \\ -1 & 2\end{array}\right)$ unit-cells were used in association with $\Gamma$-centered $4 \times 4 \times 1$ and $1 \times 4 \times 1$ k-points meshes, respectively. The plane-wave cutoff was increased to $450 \mathrm{eV}$.

Even if dispersion effects can be important to understand water bilayer stability over ice, ${ }^{13}$ we have not included them for the calculations presented in this paper. As shown by Carrasco et al. ${ }^{13}$ the main effect of including Van der Waals interactions is to increase the metal-water interaction (compared to pure BPE functional). While this total stabilization is important (about $150 \mathrm{meV} /$ water) it is similar for different water layer structures even on very different surfaces (within $30 \mathrm{meV}$ ). Therefore the effect of the Van der Waals interactions on the energy difference between the H-up and H-down phases on the same $\mathrm{Pt}(111)$ surface, is expected to be negligible over the strong electrochemical effects discussed in this study. Their global effect on the surface electrochemical stability diagram should therefore be negligible.

As shown in Fig. 1, two structures were considered: the $\mathrm{H}$-up structure with one $\mathrm{H}_{2} \mathrm{O}$ parallel to the surface (hereafter denoted $\mathrm{H}_{2} \mathrm{O}_{\text {up-flat }}$ ) and another $\mathrm{H}_{2} \mathrm{O}$ with an $\mathrm{O}-\mathrm{H}$ bond pointing toward the vacuum (hereafter denoted $\mathrm{H}_{2} \mathrm{O}_{\text {up }}$ ); and the $\mathrm{H}$-down structure with one $\mathrm{H}_{2} \mathrm{O}$ parallel to the surface (hereafter denoted $\mathrm{H}_{2} \mathrm{O}_{\text {down-flat }}$ ) and another $\mathrm{H}_{2} \mathrm{O}$ showing an $\mathrm{O}-\mathrm{H}$ bond pointing toward the $\mathrm{Pd}$ surface (hereafter denoted $\mathrm{H}_{2} \mathrm{O}_{\text {down }}$ ). Many different approaches were proposed in the literature to model electrochemical effects on surface. ${ }^{29-33}$ Among them, the approach initially proposed by Filhol and Neurock ${ }^{2,34}$ and lately improved by Mamatkulov and Filhol ${ }^{23}$ proposes to model electrochemical effects by charging the unit cell and compensating this charge by an homogeneous background. As described in previous papers, ${ }^{2,23,34}$ a total charge excess $Q_{t}$ is added to the unit cell which splits onto the two surfaces of the slab. Furthermore, as presented in Ref[23] because of the compensating background, the effective surface charge $q$ for one surface is equal to half the fraction of vacuum size over the total unit cell size in the z-direction times the total charge $Q_{t}$ (i.e. $q=0.264 Q_{t}$ ). The associated new correction reduces the calculated system capacitance in comparison to the previous 
correction that tends to overestimate it and then over-stabilize the charged systems. Furthermore, it allows recovering the correct relation between the charged-induced electric field at the surface and the surface electrochemical free energy. In many cases the effect should be quantitatively moderate (vide infra) but in some other ones (large metal slab, strong surface polarization ...) the new correction should improve drastically the results. Then, the new correction gives energies for charged systems that are more reliable.

The electrochemical potential $\mu$ of the system is referenced to the vacuum potential in the middle of the inter-slab vacuum. Therefore, we define the electric potential of the surface $V($ in $V$ ) as the opposite of the electrochemical potential $\mu$ (in eV): $V=-\frac{\mu}{e} . \mathrm{V}$ is also numerically equal to the surface work function $\phi$ (in eV). Following the latest correction for background, the electrochemical free energy for one water-Pd surface in the unit-cell writes accordingly ${ }^{23}$ :

$$
F=\left(E_{d f t}\left(Q_{t}\right)-E_{d f t}(0)+\int_{0}^{Q_{t}}\left\langle V_{T}\left(Q_{t}\right)\right\rangle d Q_{t}\right) \times 0.264+\frac{1}{2} E_{d f t}(0)+\frac{q \mu}{e}
$$

where $\mathrm{E}_{\mathrm{DFT}}$ is the DFT energy, $\left\langle V_{T}\left(Q_{t}\right)\right\rangle$ is the average self-consistent potential in the charged supercell, $\mu$ is the electronic potential of the slab referenced to the vacuum level and $\mathrm{e}$ is the absolute value of the electron charge (i.e. $e=1.60210^{-19} \mathrm{C}$ ). To compare this potential scale referenced to the vacuum to the more usual standard hydrogen electrode (SHE) reference, one can use the estimated conversion relation: $V_{\text {SHE }}=V_{\text {vacuum }}-4.6 .^{35-38}$

\section{Evolution of water structures with potential}

First, we focus on two model structures for the water monolayer on the $\mathrm{Pd}(111)$ surface, namely $\mathrm{H}$-up and $\mathrm{H}$-down. Both $\mathrm{H}$-up and $\mathrm{H}$-down structures were studied in a potential range from 1 to $8 \mathrm{~V}$. However, for a potential larger than $6 \mathrm{~V}$, the $\mathrm{H}$-down phase becomes unstable and spontaneously switches to H-up. For both phases, a strong variation of the water molecules distance to the surface is observed as a function of the applied potential. Interestingly, the variation is significantly different depending on the phase and the water molecule considered.

\section{A) Flat water}

For both $\mathrm{H}$-up and $\mathrm{H}$-down structures, the O-Pd distance between the surface and the $\mathrm{H}_{2} \mathrm{O}_{\text {flat }}$ molecule strongly contracts with the potential increase, from about $4 \AA$ to $2.4 \AA$, consistently with an adsorption of the molecule on the surface (see Fig. 2). Interestingly, this contraction starts for a potential higher than $2.8 \mathrm{~V}$ for $\mathrm{H}_{2} \mathrm{O}_{\text {up-flat }}$ and higher than $3.9 \mathrm{~V}$ for $\mathrm{H}_{2} \mathrm{O}_{\text {down-flat. }}$ In contrast to our 
previous study on the $\mathrm{CO} / \mathrm{Pt}(111)^{23}$ interface, this behavior cannot easily be explained by pure electrostatic effects. Indeed, since the $\mathrm{H}_{2} \mathrm{O}_{\text {flat }}$ molecule dipole is here quasi-parallel to the surface, no efficient electrostatic interaction can occur with the orthogonal electric field induced by the surface charging. Hence, in the present case of $\mathrm{H}_{2} \mathrm{O}_{\text {flat }} / \mathrm{Pd}(111)$, electrostatic effects should not dominate the structural response to external potential of the $\mathrm{H}_{2} \mathrm{O}$ monolayer, in contrast with the $\mathrm{CO} / \mathrm{Pt}(111)$ case where the CO dipole was parallel to the electric field.

\section{B) Water down}

The $\mathrm{H}-\mathrm{Pd}$ distance between the hydrogen atom of $\mathrm{H}_{2} \mathrm{O}_{\text {down }}$ pointing toward the surface and the closest Pd atom of the surface exhibits a volcano curve as a function of the potential (see Fig. 3). At low potential (around $1 \mathrm{~V}$ ), the Pd-H distance is weak (about $2.2 \AA$ ) and could be consistent with some $\mathrm{Pd}-\mathrm{H}$ bonding. As the potential increases, the $\mathrm{Pd}-\mathrm{H}$ distance increases and stabilizes to $2.4 \AA$ in the range of $3-4 \mathrm{~V}$ prior to decrease to $2.1 \AA$ above $4 \mathrm{~V}$. Above $6 \mathrm{~V}$, the phase becomes unstable and the $\mathrm{H}_{2} \mathrm{O}_{\text {down }}$ no longer exists. The distance shortening at low potential could be partially caused by the electrostatic $\mathrm{O}-\mathrm{H}$ dipole interaction with the negatively charged surface. However, since the H-down surface remains negative up to $5 \mathrm{~V}$, this dipole-charge interaction does not explain the volcano curve observed.

Therefore, in contrast with the CO/Pt case previously studied where the structural changes upon charging were mainly associated with the electrostatic interactions between the adsorbed molecule dipole and the charge induced electric field, for the water-Pd(111) case, modifications of the chemical bonding should be the leading effect.

\section{Electronic interaction rationalization}

To further understand the electronic interaction that tunes the Pd-water bonding, a classical molecular orbital approach was used.

\section{A) $\mathrm{H}_{2} \mathrm{O}_{\text {flat }}$ interaction with the Pd surface}

In the case of $\mathrm{H}_{2} \mathrm{O}_{\text {up-flat }}$ and $\mathrm{H}_{2} \mathrm{O}_{\text {down-flat, }}$ the local symmetry imposes the dominant $\sigma$-type interaction to be between the $1 b_{1}$ water orbital and the $P d \mathrm{dz}^{2}$ band (see Fig.4). Upon reduction (excess of electron), the $\mathrm{dz}^{2}$ band shifts up to higher energies due to the increase of electron-electron repulsion (adding 0.1 electron/unit cell to the surface shifts up the Fermi Level by about $0.75 \mathrm{eV}$ ). Since the $\mathrm{Pd} d$-band is nearly fully filled, the interaction with the low lying $1 \mathrm{~b}_{1}$ orbital corresponds to a classical 2-orbitals-4-electrons type repulsive interaction: In the classical molecular orbital picture this would create a total Pd-water system with both fully-filled bonding and fully-filled antibonding 
states that would be less stable than the two separated systems. As a consequence to this repulsive interaction, the $\mathrm{H}_{2} \mathrm{O}_{\text {flat }}$ molecules flee away the surface. Then, for low potential, the dominant interactions between the $\mathrm{H}_{2} \mathrm{O}_{\text {flat }}$ molecule and the Pd surface are repulsive (steric) coherently with the long Pd-O distances observed: at this potential the adsorption is essentially controlled by physisorption.

When the potential is tuned to more oxidizing conditions (by removing electrons from the system), the $d z^{2}$ band is stabilized due to a decrease of the electron-electron repulsion. The $1 b_{1}$ orbital energy of water gets then closer to the metal Fermi level: the interaction of the $\mathrm{H}_{2} \mathrm{O}_{\text {flat }} 1 \mathrm{~b}_{1}$ orbital with the filled $\mathrm{dz}^{2}$ states lying just below the Fermi level becomes strong enough to push some O-Pd anti-bonding states above the Fermi Level. Electron transfer thus occurs from these O-Pd antibonding states to the metal bands through self consistent effects. This results in filling O-Pd bonding orbitals and partially emptying $\mathrm{Pd}-\mathrm{O}$ anti-bonding orbitals: the closer the $1 \mathrm{~b}_{1}$ is to Fermi Level, the more O-Pd anti-bonding orbitals get empty, and the more bonding is the Pd-O interaction. This leads to a bond formation between $\mathrm{H}_{2} \mathrm{O}_{\text {flat }}$ and the Pd surface under oxidative conditions. As part of the electrons in the anti-bonding Pd-O orbital is transferred away, both the Pd atom and the overlying oxygen atom should loose electron density, thus corresponding to an oxidative adsorption of water on Pd.

A useful tool for illustrating this mechanism is the Fukui function. ${ }^{39}$ The Fukui function is defined as:

$$
f(\vec{r})=\left(\frac{\partial \rho(\vec{r})}{\partial N}\right)
$$

where $\rho(\vec{r})$ is the electron density of the system and $\mathrm{N}$ is the number of electrons in the system. In the present case of a metallic surface, no gap is present in the Pd electronic structure so that the Fukui function is unique $\left(f^{+}=f^{-}=f\right)$. One should note that this function is mainly positive, but can present locally negative density contributions. The distribution $q . f(\vec{r})$ gives the spatial density of an added (or withdrawn) charge q on the surface, consistently with the orbital analysis.

The Fukui function for the $\mathrm{H}$-up and $\mathrm{H}$-down phase are given in Fig. 5 . For both cases, the $\mathrm{H}_{2} \mathrm{O}_{\text {flat }}$ present similar Fukui functions. Upon oxidation, the electron depletion is localized onto $\mathrm{adz}^{2}$-like orbital of the $\mathrm{Pd}$ atoms located on the surface and a $1 \mathrm{~b}_{1}$-like orbital of the water molecule. An increase of the electronic density is observed in between the $\mathrm{O}$ of the $\mathrm{H}_{2} \mathrm{O}_{\text {flat }}$ and the underneath $\mathrm{Pd}$ atom. This Fukui function is fully coherent with the orbital analysis discussed above with anti-bonding $d z^{2}-1 b_{1}$ orbitals shifting up above the Fermi level. The resulting charge transfer thus leads to the 
formation of a bonding O-Pd interaction characterized by the increase of the electronic density in between the two interacting atoms, although the electronic density of the system globally decreases.

\section{B) $\mathrm{H}_{2} \mathrm{O}_{\text {down }}$ interaction}

The $\mathrm{H}_{2} \mathrm{O}_{\text {down }}$ interaction with the Pd surface is more complex as the $1 b_{2}, 3 a_{1}, 4 a_{1}{ }^{*}, 2 b_{2}{ }^{*}$ water orbitals have the proper local symmetry to interact with the $\mathrm{dz}^{2}$ band of the underneath $\mathrm{Pd}$ atom. The $3 \mathrm{a}_{1}$ orbital has the weakest contribution on the hydrogen atom and is therefore neglected. The $1 b_{2}$ orbital could have a similar $4 \mathrm{e}$ - interaction with the $\mathrm{dz}^{2}$ band than the one discussed above for $\mathrm{H}_{2} \mathrm{O}_{\text {flat. }}$. However, since this orbital is lying far deeper in energy than the $\mathrm{dz}^{2}$ orbitals close to Fermi level, this interaction is negligible, even under oxidative conditions. Therefore, only the empty antibonding $4 \mathrm{a}_{1}{ }^{*}$ and $2 \mathrm{~b}_{2}{ }^{*}$ orbitals (for the $\mathrm{O}-\mathrm{H}$ bond) can efficiently interact with the $\mathrm{Pd} \mathrm{dz}^{2}$ band. The interaction is a classical 2-orbitals-2-electrons interaction with a strong $\sigma$-character. For such interaction, only the bonding orbitals are filled with electrons, the anti-bonding ones being mostly empty, which is stabilizing for the system. The qualitative orbital interaction scheme under oxidative and reductive potentials is given in Fig. 6 . The more reductive the conditions are, the closer are the filled $\mathrm{dz}^{2}$ band and the empty $4 \mathrm{a}_{1}{ }^{*}-2 \mathrm{~b}_{2}{ }^{*}$ orbitals, and the more efficient the Pd-H bonding interaction (bond strengthening) is. Upon reducing conditions, the formation of a $\mathrm{Pd}-\mathrm{H}$ bond is then expected and should weaken as the conditions turn to oxidation. This is consistent with the short $\mathrm{Pd}-\mathrm{H}$ distance $(2.1 \mathrm{~A})$ observed at low potential and the moderate increase with increasing potential.

Under reductive conditions, the associated Fukui function (see Fig. 5) show the charging (in blue) of the $P d \mathrm{dz}^{2}$-like orbital and the mixing of the $4 a_{1}{ }^{*}$-like and $2 b_{2}{ }^{*}$-like orbitals. Since there is an important increase of the electronic density in between the oxygen atom and the underneath $\mathrm{Pd}, \mathrm{a}$ bonding behavior is expected for reductive conditions as exposed in the electronic interaction analysis (vide supra). Losses of electronic density (in yellow) are also observed mainly on the $\mathrm{H}$ pointing toward the surface. This is partially caused by the electric field induced polarization that tends to weaken the charge of the hydrogen atom pointing toward the negatively charged surface; but it is also the consequence of indirect self-consistent effects: since the charging also increases the charge on the oxygen $\mathrm{p}_{\mathrm{z}}$ orbital of the $\mathrm{H}_{2} \mathrm{O}_{\mathrm{flat}}$, this orbital is destabilized which modifies its interaction with the surrounding $\mathrm{H}$ atoms. This phenomenon induces some charge reorganization and a significant loss of electron density on the hydrogen atom.

At this stage, neither the orbital interactions nor the shape of the Fukui functions nor the electrostatic interactions can explain why the $\mathrm{Pd}-\mathrm{H}_{2} \mathrm{O}_{\text {down }}$ interaction decreases under oxidizing conditions above $4 \mathrm{~V}$. Indeed, in this potential range, the 2-electrons interaction weakens and the electron density in between the $\mathrm{O}$ and $\mathrm{Pd}$ atoms decreases. This should lead to a weakening of the 
$\mathrm{Pd}-\mathrm{H}$ bond and therefore an increase of the distance whereas a decrease is observed. Furthermore, from the electrostatic point of view, it is expected that the $\mathrm{OH}$ dipole interacts with a more and more positive surface in more and more repulsive way. Eventually, although the $1 b_{2}-d_{z} 2$ orbital interaction could contribute to some decrease of the $\mathrm{Pd}-\mathrm{H}$ distance, this interaction should be considered negligible even at a high potential.

To rationalize this surprising behavior, it is important to pay attention to the $\mathrm{H}_{2} \mathrm{O}_{\text {flat }}$ behavior. As observed in Fig.2 and Fig. 3, the $\mathrm{Pd}-\mathrm{H}_{2} \mathrm{O}_{\text {down }}$ distance decrease is concomitant with the water flat oxidative adsorption. As $\mathrm{H}_{2} \mathrm{O}_{\text {down }}$ and $\mathrm{H}_{2} \mathrm{O}_{\text {flat }}$ are strongly linked by $\mathrm{H}$-bonds, the $\mathrm{H}_{2} \mathrm{O}_{\text {flat }}$ strong adsorption under oxidation is also pushing down the $\mathrm{H}_{2} \mathrm{O}_{\text {down }}$ toward the surface causing the distance shortening, and strongly increasing the steric repulsion between the surface and the $\mathrm{H}_{2} \mathrm{O}_{\text {down }}$. For a large enough potential, the steric repulsion becomes too strong and the $\mathrm{H}_{2} \mathrm{O}_{\text {down }}$ flips up to $\mathrm{H}_{2} \mathrm{O}_{\text {up }}$. This explains the transformation of the $\mathrm{H}$-down phase into the $\mathrm{H}$-up for potential higher than $6 \mathrm{~V}$.

This study shows that the structural response to oxidizing/reducing conditions of water molecules adsorbed on a metal surface can be rationalized using qualitative molecular orbitals analysis. Electrochemical phenomena induce a modification of the orbital interactions by shifting the energy of the metal d-band relatively to the orbitals of the $\mathrm{H}_{2} \mathrm{O}$ adsorbate. Furthermore, the modification of the electronic interactions is clearly linked to the shape of the Fukui function that is a direct, easy-handle and useful tool to understand the electrochemical reactivity of a system.

\section{Revised electrochemical stability diagram for $\mathrm{H}_{2} \mathrm{O} / \mathrm{Pd}(111)$}

\section{A) Stability diagram}

A revised stability diagram for the $\mathrm{H}$-up vs. $\mathrm{H}$-down phases has been computed following the approach reported in Ref $\left[{ }^{23}\right]$ and is given in Fig. 7. In this approach, a correction is added to get rid of one drawback of the initial homogeneous background method: in the initial method, the surface charge is overestimated due to volume charge trapping by the background. In the present case of water-Pd, it leads to surface charges being 0.527 times than the charges computed in the previous method. It should be noted however, that the potential limit for the $\mathrm{H}$-up and $\mathrm{H}$-down relative stability is only slightly shifted by $0.2 \mathrm{~V}$ compared to our previous work whose main conclusions remain valid. The $\mathrm{H}$-down phase is stable at potential lower than $4.5 \mathrm{~V}$. For higher potential, the $\mathrm{H}$ up phase is the most stable. At $4.5 \mathrm{~V}$, a two-phase equilibrium is reached following:

$$
H_{u p}^{0.12+}+0.26 e^{-} \leftrightarrow H_{d o w n}^{0.14-}
$$


At equilibrium, the amount of electron exchange per surface area for this reaction is nearly one order of magnitude larger than the one found for $\mathrm{CO} / \mathrm{Pt}(111) .{ }^{23}$ This clearly shows that whereas the $\mathrm{CO} /$ $\mathrm{Pt}(111)$ interface is mainly governed by electromechanical phenomena, $\mathrm{H}_{2} \mathrm{O} / \mathrm{Pd}(111)$ is an intrinsic electrochemical system associated with a strong charge exchange between the two $\mathrm{H}$-up and $\mathrm{H}$ down phases, as the equilibrium potential is reached. Furthermore, the neutral $\mathrm{H}$-up and H-down structures remain metastable and the equilibrium neutral structure is therefore a mixture of $54 \%$ positively charged $H_{u p}^{0.12+}$ and $46 \%$ negatively charged $H_{\text {down }}^{0.14-}$.

As previously shown, ${ }^{23}$ the second order expansion of the energy can be expressed as :

$$
E=E_{0}+C V_{0}\left(V-V_{0}\right)+\frac{1}{2} C\left(V-V_{0}\right)^{2}
$$

where $\mathrm{C}$ is the system capacitance and $\mathrm{V}_{0}$ is the potential associated with the neutral phase. In this limit, the free electrochemical energy is:

$$
F=E-q V=E_{0}+C V_{0}\left(V-V_{0}\right)+\frac{1}{2} C\left(V-V_{0}\right)^{2}-C V\left(V-V_{0}\right)=E_{0}-\frac{1}{2} C\left(V-V_{0}\right)^{2}
$$

It shows a parabola shape, with a maximum for the potential of zero charge $\mathrm{V}_{0}$. This is qualitatively observed for H-up and $\mathrm{H}$-down phases in Fig. 7. Nevertheless, both $\mathrm{H}$-Up and $\mathrm{H}$-down free energy curves are highly non-parabolic, in contrast to what was previously found for $\mathrm{CO} / \mathrm{Pt}(111){ }^{23}$ This highlights the importance of higher-order terms in the series expansion (eqn. 1). The average surface capacitance for the H-down and H-up phases is $9.1310^{-2} \mathrm{~F} \cdot \mathrm{m}^{-2}$ and $7.0210^{-2} \mathrm{~F} \cdot \mathrm{m}^{-2}$, respectively. Hence, even if the inter-slab vacuum layer is the same in both computed phases, the capacitance differs by $20 \%$ between the two phases. This disparity can be directly explained using the Fukui functions of Fig. 5 as the spatial distribution of the added charge in the two structures ( $\mathrm{H}$-up and $\mathrm{H}$ down) is different. For H-down case, the added charged distribution (given by the Fukui function) has a slightly larger expansion that should allow a reduced self-repulsion and is furthermore stabilized by dipolar interaction with the $\mathrm{H}_{2} \mathrm{O}_{\text {down }} \mathrm{O}-\mathrm{H}$ dipole: this allows increasing the $\mathrm{H}$-down phase capacitance. The capacitance is then not only linked to the global surface shape (curvature radius or condenser geometry) but also to the adsorbate's geometry that could enhance the stabilization of the added charge. This is confirmed by the normalized capacitance of the water monolayer which is here twice the one found for the CO/Pt(111) case. In CO/Pt(111), the Fukui function of the surface is mainly localized on the oxygen atom of the CO molecule: this leads to an enhanced self repulsion of the added charge and to a weakening of the surface capacitance, therefore reducing the ability of the surface to be charged. The water monolayer on Pt(111) is then a case of high capacitive interface 
with highly charged surfaces associated with a non-parabolic shape of the free energy. This suggests that $\mathrm{H}_{2} \mathrm{O} / \mathrm{Pt}(111)$ system meets all criteria ${ }^{23}$ to be dominated by electrochemical effects rather than by electromechanical effects.

The phase stability diagram predicts that the stable neutral water monolayer is in reality a mixture of charged domains because of strong electrochemical effects. These effects that were not taken into account in previous studies to determine the water monolayer structure could indeed be important hidden parameters.

\section{B) The neutral water-Pd(111) surface}

Although water-Pd surfaces are known to undergo complex structures from both experimental $\left.\right|^{9,18,40,41}$ and theoretical investigations, ${ }^{6,9,18,21}$ few remaining questions still deserve to be addressed. First, the structure close to the monolayer proposed initially by Cerda et al. were not more stable than the $\mathrm{H}$-down phase and no driving force was given for its stabilization. ${ }^{9}$ Second, at one monolayer coverage (i.e. before the 3D-growth starts), the experimental STM images of the $\mathrm{H}_{2} \mathrm{O}$ Pd surface show bright stripes of water structures surrounded by large dark zones ${ }^{9,18}$ that cover a large fraction of the total surface area (more than 25-30\%). These dark zones were considered as free of water by the authors. This suggests that either full water monolayers cannot be achieved due to strong repulsion between water domains, or part of the water molecules is invisible to the STM tip.

Our model suggests that a neutral surface built on a mixture of nearly 50/50 $\mathrm{H}$-up and $\mathrm{H}$ down domains with opposite charges stabilizes the system by at most $40 \mathrm{meV}$ (per water dimer). This has to be compared to the neutral $\mathrm{H}$-down which is already about $80 \mathrm{meV}$ more stable than the $\mathrm{H}$ up. It is worth noting, however, that our electrochemical model assumes the high capacitance limit ${ }^{23}$ which may not be always relevant for a UHV experiments as it may over-stabilize charged phases. To address this question and validate our approach, we performed supercell calculations on a neutral 135-atoms slab made of symmetrically adsorbed $n \mathrm{H}$-up and (5-n) $\mathrm{H}$-down subunits (with $n=0 . .5$ ). The relative energy for this structure is given in Fig. 8 . It shows that the mixed structure for $n=1$ and 2 is more stable than the pure $\mathrm{H}$-down phase by $20 \mathrm{meV} / \mathrm{sub}$ unit (half the maximum energy predicted (vide supra)); for $\mathrm{n}=3$, the mixed structure is only $7 \mathrm{meV} / \mathrm{sub}$ unit less stable than the H-down phase, although it contains a majority of $3 \mathrm{H}$-up sub-units which are far less stable than $\mathrm{H}$-down sub-units. We then studied the excess energy $E_{e x c}$ of the mixed system compared to the neutral and independent $\mathrm{H}$-up to $\mathrm{H}$-down pure phases associated with the equation:

$$
n \cdot \mathrm{H}_{2} \mathrm{O}^{0}(\text { up })+(5-n) \cdot \mathrm{H}_{2} \mathrm{O}^{0}(\text { down }) \leftrightarrow\left(n \cdot \mathrm{H}_{2} \mathrm{O}^{\delta+}(\text { up }),(5-n) \cdot \mathrm{H}_{2} \mathrm{O}^{\delta-}(\text { down })\right) \quad E_{\text {exc }}
$$


This excess energy removes the intrinsic chemical components ${ }^{42}$ of both $\mathrm{H}$-up and $\mathrm{H}$-down phases and should mainly be the balance between the mechanical strain caused by the mixing of structures and the predicted stabilization due to electrochemical effects. This excess energy is found to be between -36 and -40 meV for $n=1,2,3$. While it increases rapidly for higher $n$, it remains negative for any composition, showing that the mixture of charge $\mathrm{H}$-up and $\mathrm{H}$-down surfaces is always favored over two independent neutral phases. The mixing effect is then maximum for $n=2$, and since the $\mathrm{H}$ up phase is far less stable than the $\mathrm{H}$-down, the absolute stability is shifted to a lower $\mathrm{n}$ with less $\mathrm{H}$ up $(n=1)$.

The origin of this enhanced stabilization can be found in the variation of the electronic density upon water adsorption, $\rho_{\text {var }}$, in a frozen geometry (i.e. $\rho_{\text {var }}=\rho_{P d+\text { water }}-\rho_{P d}-\rho_{\text {water }}$ ). This is presented in Fig. 9 for various $n$. The charge variation obtained for the pure $H$-up phase $(n=5)$ confirms our orbital analysis by showing that the $\mathrm{H}$-up bonding is dominated by the $\mathrm{H}_{2} \mathrm{O}_{\text {flat }}$ adsorption through $1 b_{1}$ $\mathrm{dz}^{2}$ 4-electrons-type interaction. This interaction is associated with an electron loss on the $\mathrm{H}_{2} \mathrm{O}_{\text {flat }}$ and the underneath $\mathrm{Pd}$ atom, as confirmed by the respective $+0.003 \mathrm{e}$ and $+0.005 \mathrm{e}$ Bader charges computed, and by the electron transfer toward other Pd atoms of the surface (Bader charge of $0.05 \mathrm{e})$. As the uncharged structure has a low potential of $2.8 \mathrm{~V}$, this interaction is weak, explaining the low adsorption energy of $\mathrm{H}$-up phase. The $\mathrm{H}_{2} \mathrm{O}_{\text {up }}$ molecule in this phase appears to be electronically inactive.

The charge variation obtained for the pure H-down phase $(n=0)$ shows that both $\mathrm{H}_{2} \mathrm{O}_{\text {flat }}$ and $\mathrm{H}_{2} \mathrm{O}_{\text {down }}$ interact strongly with the surface. The $\mathrm{H}_{2} \mathrm{O}_{\text {flat }}$ exhibits a 4-electrons repulsive $1 \mathrm{~b}_{1}-\mathrm{dz}^{2}$ interaction $\left(\mathrm{H}_{2} \mathrm{O}_{\text {flat }}\right.$ charge is $0.03|\mathrm{e}|$ and the underneath $\mathrm{Pd}$ is $\left.0.08|\mathrm{e}|\right)$; and the $\mathrm{H}_{2} \mathrm{O}_{\text {down }}$ a $2 \mathrm{~b}_{2}-3 \mathrm{a}_{1}-\mathrm{dz}^{2}$ stabilizing interaction associated with an electron density increase between $\mathrm{H}_{2} \mathrm{O}_{\text {down }}$ and the $\mathrm{Pd}$ (whose Bader charge is $-0.08|e|$ ). Since the potential is high for this structure (about $5 \mathrm{~V}$ ), the $1 b_{1}-\mathrm{dz}^{2}$ interaction is stronger than for the $\mathrm{H}$-up phase (vide supra), although not optimal for the $\mathrm{H}_{2} \mathrm{O}_{\text {down }}-\mathrm{Pd}$, one which should be maximal for lower potential. Nevertheless, the interaction is stronger than for independent $\mathrm{H}$-up than for $\mathrm{H}$-down as both interactions are active in the mixed phase, leading to an enhanced stability of $80 \mathrm{meV} /$ sub-unit.

This interaction is further increased by $20 \mathrm{meV}$ if one $\mathrm{H}_{2} \mathrm{O}_{\text {down }}$ molecule is flipped to an $\mathrm{H}_{2} \mathrm{O}_{\text {up }}$ configuration (Fig. 9, $n=1$ ). The charge density variation shows that the two stabilizing $\mathrm{H}_{2} \mathrm{O}_{\text {flat }}-\mathrm{Pd}$ and one $\mathrm{H}_{2} \mathrm{O}_{\text {down }}-\mathrm{Pd}$ interactions are lost. But, the closest $\mathrm{H}_{2} \mathrm{O}_{\text {flat }}$ to the perturbation shows an increased depletion of its electron density (the water charge being $0.045 \mathrm{e}$ and the underneath $\mathrm{Pd} 0.11 \mathrm{e}$ ) suggesting a stronger oxidant adsorption. This increased charge depletion on the $\mathrm{H}_{2} \mathrm{O}_{\text {flat }}$ is stabilized by the water dipole of the adjacent $\mathrm{H}_{2} \mathrm{O}_{\text {up }}$. A charge density increase in between all the $\mathrm{H}_{2} \mathrm{O}_{\text {down }}$ and 
the surface ( $P d$ charges being between -0.08 and $-0.12|e|$ ) is also observed. This suggests that some of the $\mathrm{H}_{2} \mathrm{O}_{\text {flat }}$ and all the $\mathrm{H}_{2} \mathrm{O}_{\text {down }}$ bonds with the Pd surface are getting stronger. This is confirmed by the shortening of two $\mathrm{H}_{2} \mathrm{O}_{\text {flat }}-\mathrm{Pd}$ bond from $2.67 \AA$ for $\mathrm{n}=0$ to 2.50 and $2.40 \AA$ for $n=1$. The average geometry around $\mathrm{H}_{2} \mathrm{O}_{\text {down }}$ is coherent with the distances found for the model (see Fig. 3 ) for this $\mathrm{n}=1$ surface potential $(4.88 \mathrm{~V})$.

The structure $\mathrm{n}=2$ is more easy to decompose into two sub-part. While the bonding between $\mathrm{H}_{2} \mathrm{O}_{\text {flat }}$ and the surface is lost in the $\mathrm{H}$-down part (where only $\mathrm{H}_{2} \mathrm{O}_{\text {down }}-\mathrm{Pd}$ bonds still exist), the $\mathrm{H}_{2} \mathrm{O}_{\text {flat }}-\mathrm{Pd}$ bonding is strong in the H-up part. The H-up sub-units are globally electron depleted and associated with a charge of +0.118 e while the $\mathrm{H}$-down sub-units are electron enriched with a global charge of 0.123e. The geometry of the two parts is consistent with the one found in Figs. 2 and 3 for the potential corresponding to the $\mathrm{n}=2$ surface $(4.53 \mathrm{~V})$ with average $\mathrm{H}_{2} \mathrm{O}_{\text {flat }}-\mathrm{Pd}$ and $\mathrm{H}_{2} \mathrm{O}_{\text {down }}-\mathrm{Pd}$ distances around $2.45 \AA$ and $2.35 \AA$, respectively. This structure is consistent with the equilibrium structure predicted from the electrochemical phase stability diagram with both the predicted potential and the local charging of the sub-units. It is however not the most stable surface: this was expected as the model considers ideal infinite separated $\mathrm{H}$-up and $\mathrm{H}$-down phases and does not take into account the cost of structural and electronic distortions that occur at interfaces between the two phases. Nevertheless, this surface $(n=2)$ remains more stable than the H-down phase and exhibits the highest excess energy suggesting that electrochemical effects are essential to understand its organization. Furthermore, as the sub-units charges and potential of this interface are coherent with the ones given by the electrochemical model, the large capacitance approximation is certainly appropriate for such a system, thus validating our approach.

The structure corresponding to $n=3,4$ remains more stable than the $\mathrm{H}$-up phase as the slightly more oxidizing surface potential (resp. 3.75 and 3.39V) allows a better adsorption of the $\mathrm{H}_{2} \mathrm{O}_{\text {flat }}$ than in the pure $\mathrm{H}$-up case $(2.78 \mathrm{~V})$.

As the surface potential shifts from $5 \mathrm{~V}$ to $2.78 \mathrm{~V}$ with $\mathrm{n}$, we can check on these neutral cells the validity of the electrochemical analysis (vide infra). Each $\mathrm{H}$-up/H-down subunit of these neutral cells can be compared with the associated electrochemical model at the associated potential. With the potential decrease, the $\mathrm{H}_{2} \mathrm{O}_{\text {flat }}-\mathrm{Pd}$ bond weakens as seen by the larger 4-electrons repulsive $1 b_{1}-\mathrm{dz}^{2}$ interaction (associated with a smaller negative isosurfaces and lower Bader charge) and $\mathrm{H}_{2} \mathrm{O}_{\text {flat }}-\mathrm{Pd}$ bond increases as the $2 b_{2}-3 a_{1}-d z^{2}$ stabilizing interaction is stronger (larger isosurfaces and Barder charges): the $\mathrm{H}$-up/H-down subunits behave similarly to what is predicted by the electrochemical approach. Then, for a neutral water monolayer on $\mathrm{Pd}(111)$, the formation of charged subunits is always favored over the neutral homogeneous surface as predicted by the electrochemical stability 
diagram. The stabilization of a mixed phase made of charged domains can be surprising as the resulting charge separation is usually associated with a high energy cost. In the present case we have shown that it is stabilized by the formation of strong $\sigma$-type bonds between water and the metal surface and that an electrochemical approach on small systems can model can reproduce the behavior of a far larger neutral system, where disproportionation happens creating locally charged domains.

\section{Discussion}

\section{A Surface disproportionation}

The spontaneous formation of charged domains on a neutral surface can appear surprising at first sight. It is however similar to the electrochemical disproportionation that a compound may undergo to form one reduced and one oxidized products, such as the classical dichlorine disproportionation $\left(\mathrm{Cl}_{2}+2 \mathrm{OH}^{-} \rightarrow \mathrm{H}_{2} \mathrm{O}+\mathrm{Cl}^{-}+\mathrm{ClO}^{-}\right)$. In the present case, the water can be seen as presenting both an associated oxidant, which is the positively charged $\mathrm{H}$-up phase and a reducer, which is the negatively charged H-down phase. The charged phases are stabilized because of the creation of strong bonds upon oxidation for the $\mathrm{H}$-up and reduction for the H-down. In the meanwhile, the charge splitting induces an energy cost that is linked the charge separation and the system capacitance. In this case, the local charged domains are stabilized by two electrostatic effects; the first one is the interaction of the positive charge of the $\mathrm{H}$-up with the negative charge of the $\mathrm{H}$ down: the further away these domains, the less stabilizing the electrostatic interaction (by lowering the effective capacitance); the second is the stabilization of the charge by local dipolar interactions. The dipolar interactions are more efficient in the case of the $\mathrm{H}$-down phase where the added electron density is located between the $\mathrm{H}$ of $\mathrm{H}_{2} \mathrm{O}_{\text {down }}$ and the $\mathrm{Pd}$ surface, therefore allowing an optimum charge-dipole interaction. In the $\mathrm{H}$-up phase, the $\mathrm{H}_{2} \mathrm{O}_{\text {up }}$ dipole moment can also stabilize the electronic depletion of the $\mathrm{H}_{2} \mathrm{O}_{\text {flat }}$ under oxidation, but is less efficient than for $\mathrm{H}_{2} \mathrm{O}_{\text {down }}$ since the orientation and the distance are less beneficial. The local charging of the $\mathrm{H}$-down phase is then slightly stabilized and induces a capacitance increased by $20 \%$ compared to $\mathrm{H}$-up (vide supra) .

The stability of the phenomenon with temperature is not straightforward. As the excess energy per water molecule is of the order of room temperature thermal energy, it could be expected that for a given high temperature, the thermal fluctuation would render equal $\mathrm{H}$-up and $\mathrm{H}$-down phases leading disappearance of the charged domains. Nevertheless, the interaction of these charged domains is long range through the surface potential, and therefore could lead to correlated 
fluctuations rather than random: if a fluctuation increases the amount of $\mathrm{H}$-up, the whole surface potential would shift down, thus stabilizing all the H-down phases and preventing further growth of the $\mathrm{H}$-up. Then, the surface disproportionation could be quite stable with temperature and could possibly even exist for liquid solvent at a metal interface.

\section{B Conditions for surface disproportionation}

For a surface to disproportionate, it would require two different structures with the same global stoichiometry and close energy. On the contrary, if one of the two phases is far more stable, either only the neutral form of this phase would dominates or the charging effects would be negligible. Second, the two phases should be associated with very different work functions ( $\Delta \phi$ being the difference between the two work functions) and a large charging capacitance $C$ (linked with the local surface geometry and with the formation of a capacitor created by the mixing of phases with opposite charges) since the each phase charging should vary as $q=\Delta \phi . C$. The work function is directly linked with the surface dipole. ${ }^{43,44}$ Then, all adsorbates with competitive adsorptions structures but different dipole moment (alkenes, aromatics...), as well as any polar solvent showing a different orientation at a metal interface ${ }^{45-47}$ (water, alcohol, halogeno-alkanes....) should be candidates for such a phenomena. The large capacitance can occur upon electrochemical conditions because of the presence of the double layer, for small particles and tips because of the local large curvature radius, and for strong domain mixing where the positively charged phases are close enough to the negatively charged ones to minimize the charging cost.

Then, solvents under electrochemical conditions (presence of charges salts in the solvent to create a double layer) should spontaneously undergo a surface disproportionation: for water-metal interface, H-up and H-down-like domains should both exist, associated with different local charging and different local electrochemical properties. This suggests that these domains can have a very different reactivity, catalytic and electrocatalytic properties. This was already found in previous calculations on ice- $\operatorname{Pd}(111)^{2}$ interface electrochemistry. On this interface, water can be oxidized or reduced. Under reduction, the $\mathrm{H}$-down-like structure of ice-Pd was the most reactive leading to $\mathrm{H}$ adsorbed on the surface and hydroxide anion formation while the H-up-like phase was inactive for the corresponding potential. In opposite, under oxidation, $\mathrm{H}$-up-like structure converts into an adsorbed $\mathrm{OH}$ and a hydronium cation while the $\mathrm{H}$-down is inactive. For the modeling of chemical reactivity in these domains, both the interface type and the local charging should be accounted to properly understand these systems activity.

\section{Proposition on the surface construction}


We have shown that the formation of mixed charged phases should stabilize a neutral water$\mathrm{Pd}(111)$ surface in comparison with pure neutral model phases. The phases we have calculated are certainly not the most stable, but allow to give some more constraints to the so called 2-D ice rules. ${ }^{9,18}$ These rules state that "water adopts geometries that maximize O-bonding to the metal and H-bonding to neighboring molecules". We have seen (vide supra) that the neutral H-down phase is more stable by $80 \mathrm{meV}$ than the $\mathrm{H}$-up, even if the number of O-Pd and $\mathrm{H}$-bonds are the same. This arises from the fact that $\mathrm{H}_{2} \mathrm{O}_{\text {flat }}$ only bonds efficiently with the Pd surface at high potential: to reach high potential, the total surface dipole should point toward the surface, ${ }^{43,44}$ which might occur when $\mathrm{H}_{2} \mathrm{O}_{\text {down }}$ are present. Therefore, a large part of the water molecules that are not fully $\mathrm{H}$-bonded should be in a $\mathrm{H}_{2} \mathrm{O}_{\text {down }}$ position in order to increase the surface potential and maximize the $\mathrm{H}_{2} \mathrm{O}_{\text {flat }}$ interaction with the $\mathrm{Pd}(111)$. It should be noted here that the $\mathrm{H}_{2} \mathrm{O}_{\text {down }}$ also creates $\mathrm{H}$-Pd bonds that are the strongest at lower potential. Then, the creation of negatively charge $\mathrm{H}$-down phase optimizes the $\mathrm{H}_{2} \mathrm{O}_{\text {flat }}$ interaction with the surface while positively charged $\mathrm{H}$-up optimizes the $\mathrm{H}_{2} \mathrm{O}_{\text {down }}$ interaction with the surface. Such an extended rule has been demonstrated in recent calculation ${ }^{18}$ of water cluster on $\mathrm{Pd}(111)$ and $\mathrm{Ru}(0001)$ (before water dissociation) where the large optimized clusters consists in a core of $\mathrm{H}_{2} \mathrm{O}_{\text {flat }}$ surrounded by some $\mathrm{H}_{2} \mathrm{O}_{\text {down }}$. The electrochemical rule can also help in explaining the monolayer organization observed by $\mathrm{STM}^{9,18}$ where bright island (associated with water on the surface) are separated by darker zone (associated with water-free domains). The water molecules on the border of an $\mathrm{H}_{2} \mathrm{O}_{\text {flat }}$ cluster have one $\mathrm{H}$ that is not $\mathrm{H}$-bonded with other water molecules. Shifting these $\mathrm{H}_{2} \mathrm{O}_{\text {flat }}$ into $\mathrm{H}_{2} \mathrm{O}_{\text {down }}$ allows creating $\mathrm{H}-\mathrm{Pd}$ bonds that absorb part of the surface electronic density. This should reinforce the other $\mathrm{H}_{2} \mathrm{O}_{\text {flat }}$ bonding. The centre and the border of the cluster thus become positively and negatively charged, respectively. The different islands interact through repulsive electrostatic interactions thus preventing their fusion. Furthermore, the size of these islands apart from structural stress should also be influenced by the electrochemical effects. For too large islands, the amount of $\mathrm{H}_{2} \mathrm{O}_{\text {flat }}$ becomes very large in comparison to the $\mathrm{H}_{2} \mathrm{O}_{\text {down }}$, therefore lowering the efficiency of the electrochemical stabilization. As experimentally observed the islands cannot grow over a certain size and tend to be ribbon-like in order to maximize the frontier between the occupied and empty zone to maximize the interface between the positively charged centre and the negatively charged border (increasing the island capacitance to maximize stabilizing electrostatic effects).

It should be noted that another hypothesis could be that the STM dark zone are in fact not empty. It was previously shown experimentally ${ }^{19}$ on $\mathrm{Ag}(111)$ and theoretically ${ }^{15}$ on $\mathrm{Pd}(111)$, that the STM corrugation of water on a metal surface can be extremely dependant on the STM tips bias and on the water charge state: this could lead to the nearly disappearance of the water molecule on the 
surface. In the STM experiments on $\mathrm{Pd}(111)$, more than $30 \%$ of the Pd sites appear not occupied at maximum coverage before $3 \mathrm{~d}$-growth, but other experiments ${ }^{11,40,41}$ (in particular temperatureprogrammed X-ray photoelectron spectroscopy) suggest a higher $0.9 \mathrm{ML}$ coverage for the water monolayer limit. Either the surface investigated by STM is not at maximum coverage, which is unlikely since $3 \mathrm{~d}$-growth begins just above the observed coverage, or part of the water molecules are not seen by the STM. If some water molecules are in the dark zone and not imaged by the STM, the existence of these bright and dark zones could be the consequence of the formation of charged domains that strongly mix to maximize their capacitance. The formation of mixed bright/dark zone appears to be general as it can be also found on $\mathrm{Ru}(0001)^{18}$ or $\mathrm{Pt}(111)^{48}$. The real nature of the dark phase is important to confirm: this can be done experimentally, by studying the inelastic electron tunneling spectroscopy (IETS) of water/Pd(111) surfaces that were previously theoretically predicted. ${ }^{21}$ Such technique is appropriate for the water phase presently investigated.

\section{Conclusion}

We have investigated the electrochemical effects correlated to the modification of the $\mathrm{Pd}(111)$ surface potential on model water structures. We have shown that $\mathrm{H}_{2} \mathrm{O}_{\text {flat }}$ undergo an oxidative adsorption at high potential while in the $\mathrm{H}$-down phase the $\mathrm{H}_{2} \mathrm{O}_{\text {down }}$ undergo a reductive adsorption at low potential. These phenomena can be directly understood using conventional methods of quantum chemistry based on orbital interactions: the electrochemical effect is included through the modification of the surface Pd band energy position relatively to the adsorbate orbitals. Then, as predicted by the electrochemical stability diagram for the model phases, we have shown that mixing $\mathrm{H}$-up and $\mathrm{H}$-down phases leads to a more stable structure. The explanation, in agreement with the phase diagram is that charged domains (positive for $\mathrm{H}$-up, negative for $\mathrm{H}$-down) form. This yields to an enhanced bonding of $\mathrm{H}_{2} \mathrm{O}_{\text {flat }}$ and $\mathrm{H}_{2} \mathrm{O}_{\text {down }}$ with the underneath surface, therefore stabilizing the system more than the charge separation destabilizes it. The occurrence of different charged domains can be seen as a surface disproportionation.

This surface disproportionation should occur each time a molecule adsorbed on a surface presents different structures, with close energies and different dipole moments. But at the same time a large capacitance is needed to limit the cost of the charge separation. This imposes some conditions on the local structure that should have a small radius of curvature (edge, steps, tip, nanoparticle) or that should be associated with counter-ions (double layer) or with an efficient mixing of the domains of opposite charge (present paper). The latter should be the case for most polar solvents at a metal interface and suggests that charged domains should be quite usual. 
But, charged domains should behave quite differently from purely neutral surfaces. They could possibly shift strongly the surface chemical reactivity so they could have impact in application domains like surface science, catalysis or electrocatalysis. Therefore, the electrochemical effects hidden in this type of surface disproportionation must be accounted in forthcoming studies.

Eventually we have shown that tools such as qualitative orbital interactions and quantitative Fukui functions are very powerful in rationalizing the electrochemical behavior of an interface: a strong link is here highlighted between the classical tools of conceptual DFT and electrochemistry that will be investigated in a forthcoming paper.

\section{Acknowledgement}

The authors acknowledge support from the Agence Nationale de la Recherche (ANR) through the SIRE action (ANR-06-CIS6-014-03) and the French computational resource centres IDRIS and CINES under contract 1011750. We also thank Dr. M.-L Bocquet, D. Loffreda and B. Diawara for the discussions and helpful suggestions.

\section{Figure captions}

Fig. 1: Structure of the H-down(left) and H-up(right) phases. The labels for the different water molecules are given.

Fig. 2: Evolution of the $\mathrm{Pd}-\mathrm{O}$ distance (shown in the inset) for the $\mathrm{H}_{2} \mathrm{O}_{\text {flat }}$ molecules in the $\mathrm{H}$ down and $\mathrm{H}$-up phases as a function of the surface potential.

Fig. 3: Evolution of the $\mathrm{Pd}-\mathrm{H}$ distance (shown in the inset) for the $\mathrm{H}_{2} \mathrm{O}_{\text {down }}$ molecules in the $\mathrm{H}$ down phase as a function of the surface potential.

Fig. 4: Diagram for the evolution of the main orbital interaction between an $\mathrm{H}_{2} \mathrm{O}_{\text {flat }}$ and the underneath $P d$ atom for a reducing potential $\left(V_{1}\right)$ and for an oxidant potential $\left(V_{2}\right)$.

Fig. 5: Isosurfaces of the Fukui function for the $\mathrm{H}$-up (left) and $\mathrm{H}$-down (right). The blue color corresponds to positive values whereas the yellow one corresponds to negative values.

Fig. 6: Diagram for the evolution of the main orbital interactions between an $\mathrm{H}_{2} \mathrm{O}_{\text {down }}$ and the underneath $\mathrm{Pd}$ atom for a reducing potential $\left(V_{1}\right)$ and for an oxidant potential $\left(V_{2}\right)$.

Fig. 7: Electrochemical phase stability diagram for the H-up and H-down water monolayer phases as a function of the applied potential. The filled squares correspond to the $\mathrm{H}$-up phase, while the empty ones to the H-down. No H-down phase was found stable above 6V. The lines are fourth order polynomial interpolations of the calculated data. The vertical black 
line at $4.6 \mathrm{~V}$ corresponds to the equilibrium potential of $\mathrm{H}$-up and $\mathrm{H}$-down phases. The energies are given per water dimer.

Fig. 8: Calculated relative energy and excess energy for a neutral water monolayer composed of $n \mathrm{H}$-up and (5-n) $\mathrm{H}$-down sub-units. The relative energies are referenced to the pure $\mathrm{H}$ down phase $(n=0)$ and given for a water dimer subunit.

Fig.9: Relaxed structures of the neutral surface built on $n \mathrm{H}$-up and (5-n) H-down sub-units for $n=0,1,2,5$. The isosurfaces corresponding to the variation of the electronic density with the adsorption of the water layer on the metal surface in the frozen geometry of the water-Pd relaxed surface are given. The blue color corresponds to an increase of the charge density while the yellow one to a decrease.

\section{References}

(1) Janik, M.; Taylor, C.; Neurock, M. Topics in Catalysis 2007, 46, 306-319.

(2) Filhol, J. S.; Neurock, M. Angewandte Chemie-International Edition 2006, 45, 402-406.

(3) Hodgson, A.; Haq, S. Surface Science Reports 2009, 64, 381-451.

(4) Thiel, P. A.; Madey, T. E. Surface Science Reports 1987, 7, 211-385.

(5) Feibelman, P. J. Science 2002, 295, 99-102.

(6) Michaelides, A.; Alavi, A.; King, D. A. Physical Review B 2004, 69, 113404.

(7) Meng, S.; Wang, E. G.; Frischkorn, C.; Wolf, M.; Gao, S. W. Chemical Physics Letters 2005, 402, 384-388.

(8) Meng, S.; Xu, L. F.; Wang, E. G.; Gao, S. W. Physical Review Letters 2002, 89, 176104.

(9) Cerda, J.; Michaelides, A.; Bocquet, M. L.; Feibelman, P. J.; Mitsui, T.; Rose, M.; Fomin, E.; Salmeron, M. Physical Review Letters 2004, 93.

(10) Schnur, S.; Gross, A. New Journal of Physics 2009, 11, 125003.

(11) Shavorskiy, A.; Gladys, M. J.; Held, G. Physical Chemistry Chemical Physics 2008, 10,

(12) Michael A, H. Surface Science Reports 2002, 46, 1-308.

(13) Carrasco, J.; Santra, B.; Klimes, J.; Michaelides, A. Physical Review Letters 2011, 106, 026101.

(14) Duan, S.; Wu, D. Y.; Xu, X.; Luo, Y.; Tian, Z. Q. Journal of Physical Chemistry C 2010, 114, 4051-4056.

(15) Filhol, J. S.; Bocquet, M. L. Chemical Physics Letters 2007, 438, 203-207. 066102.

(16) Li, X. Z.; Probert, M. I. J.; Alavi, A.; Michaelides, A. Physical Review Letters 2010, 104,

(17) Andersson, K.; Nikitin, A.; Pettersson, L. G. M.; Nilsson, A.; Ogasawara, H. Physical Review Letters 2004, 93, 196101.

(18) Tatarkhanov, M.; Ogletree, D. F.; Rose, F.; Mitsui, T.; Fomin, E.; Maier, S.; Rose, M.; Cerda, J. I.; Salmeron, M. Journal of the American Chemical Society 2009, 131, 18425-18434.

(19) Morgenstern, K.; Nieminen, J. The Journal of Chemical Physics 2004, 120, 1078610791.

(20) Schultz, Z. D.; Shaw, S. K.; Gewirth, A. A. Journal of the American Chemical Society 2005, 127, 15916-15922.

(21) Bocquet, M. L.; Lorente, N. J. Chem. Phys. 2009, 130, 124702.

(22) Weissenrieder, J.; Mikkelsen, A.; Andersen, J. N.; Feibelman, P. J.; Held, G. Physical Review Letters 2004, 93, 196102. 
(23) Mamatkulov, M.; Filhol, J. S. Physical Chemistry Chemical Physics 2011, 13, 7675-

7684.

(24) Perdew, J. P.; Burke, K.; Ernzerhof, M. Physical Review Letters 1996, 77, 3865-3868.

(25) Blochl, P. E. Physical Review B 1994, 50, 17953-17979.

(26) Kresse, G.; Joubert, D. Physical Review B 1999, 59, 1758-1775.

(27) Kresse, G.; Furthmuller, J. Computational Materials Science 1996, 6, 15-50.

(28) Kresse, G.; Hafner, J. Phys. Rev. B 1994, 49, 14251.

(29) Anderson, A. B.; Albu, T. V. Journal of the American Chemical Society 1999, 121, 11855-11863.

(30) Dabo, I. Ph. D. thesis, Massachusetts Institute of Technology, 2008.

(31) Lozovoi, A. Y.; Alavi, A. Physical Review B 2003, 68, 245416.

(32) Norskov, J. K.; Rossmeisl, J.; Logadottir, A.; Lindqvist, L.; Kitchin, J. R.; Bligaard, T.; Jonsson, H. Journal of Physical Chemistry B 2004, 108, 17886-17892.

(33) Otani, M.; Sugino, O. Physical Review B 2006, 73, 115407.

(34) Taylor, C. D.; Wasileski, S. A.; Filhol, J. S.; Neurock, M. Physical Review B 2006, 73, 165402

(35) Bockris, J. O. M.; Argade, S. D. J. Chem. Phys. 1968, 49, 5133-\&.

(36) Gomer, R.; Tryson, G. J. Chem. Phys. 1977, 66, 4413-4424.

(37) Reiss, H.; Heller, A. J. Phys. Chem. 1985, 89, 4207-4213.

(38) Rostamikia, G.; Janik, M. J. J. Electrochem. Soc. 2009, 156, B86-B92.

(39) Parr, R. G.; Yang, W. Journal of the American Chemical Society 1984, 106, 4049-4050.

(40) Clay, C.; Cummings, L.; Hodgson, A. Surface Science 2007, 601, 562-568.

(41) Gladys, M. J.; El Zein, A. A.; Mikkelsen, A.; Andersen, J. N.; Held, G. Surface Science 2008, 602, 3540-3549.

(42) Filhol, J. S.; Combelles, C.; Yazami, R.; Doublet, M. L. Journal of Physical Chemistry C 2008, 112, 3982-3988.

(43) Leung, T. C.; Kao, C. L.; Su, W. S.; Feng, Y. J.; Chan, C. T. Physical Review B 2003, 68, 195408.

(44) Wigner, E.; Bardeen, J. Physical Review 1935, 48, 84-87.

(45) Janik, M. J.; Taylor, C. D.; Neurock, M. J. Electrochem. Soc. 2009, 156, B126-B135.

(46) Ikeshoji, T.; Otani, M.; Hamada, I.; Okamoto, Y. Physical Chemistry Chemical Physics 2011, 13, 20223-20227.

(47) Laref, S.; Li, Y.; Bocquet, M. L.; Delbecq, F.; Sautet, P.; Loffreda, D. Physical Chemistry Chemical Physics 2011, 13, 11827-11837.

(48) Feibelman, P. J.; Bartelt, N. C.; Nie, S.; Thurmer, K. The Journal of Chemical Physics 2010, 133, 154703-7. 